By

Ezio Marchi

IMA Preprint Series \# 2199

(April 2008)

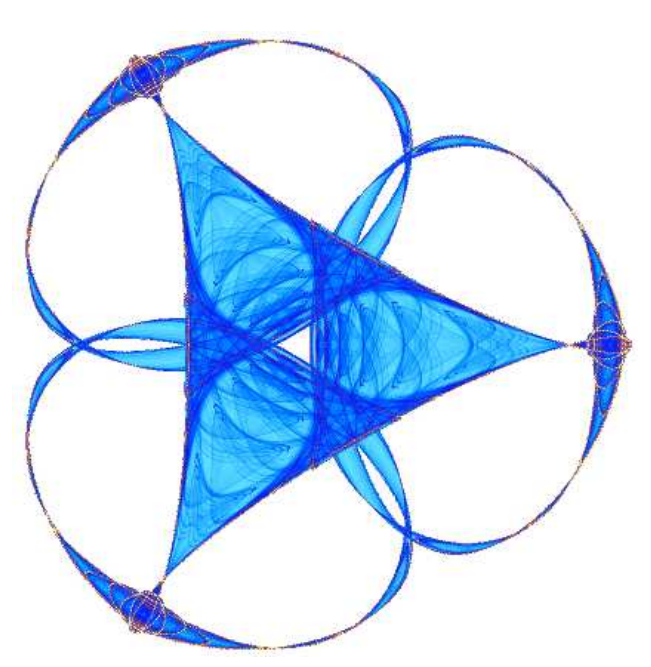

INSTITUTE FOR MATHEMATICS AND ITS APPLICATIONS

UNIVERSITY OF MINNESOTA 400 Lind Hall

207 Church Street S.E.

Minneapolis, Minnesota 55455-0436

Phone: 612-624-6066 Fax: 612-626-7370

URL: http://www.ima.umn.edu 


\title{
E-points for diagonal games II
}

by

\author{
Ezio Marchi ${ }^{* * * *}$
}

\begin{abstract}
In this paper we study and compute $E$-points in an explicit way for a special general kind of $3 k+1$ and $3 k+2$ players.
\end{abstract}

Key words: $E$-points, computation, equilibrium, non-cooperative games.

\footnotetext{
*) Founder and First Director, Instituto de Matemática Aplicada, San Luis, CONICET and Universidad Nacional de San Luis, San Luis, Argentina. Visiting the University of Barcelona, Barcelona, Spain. e-mail: emarchi@sinectis.com.ar

$\left.{ }^{* *}\right)$ This paper has been written in the Department of Applied Mathematics and Analysis, University of Barcelona, Barcelona, Spain, during a visit supported by the Dirección General de Investigaciones Científicas y Técnicas (DGIGYT) of Spain. The author acknowledges the hospitality of the Department.
} 


\section{Introduction}

We have begun to compute and study $E$-points for diagonal games in Marchi (2004) recently. We have done this for simple games. In this paper which is the continuation of the quoted one we compute and study $E$-points for a more complicated games.

With the notation of Marchi (2004) we have in general for the expected functions $E_{i}$ for player $\mathrm{i} \in \mathrm{N}=\{1, \ldots, \mathrm{n}\}$ and $\mathrm{d}(\mathrm{i}) \subset \mathrm{N}$ the set of friends of player $\mathrm{i} \in \mathrm{N}$. An $E$-point is a point $\overline{\mathrm{x}}=\left(\overline{\mathrm{x}}_{1}, \ldots, \overline{\mathrm{x}}_{\mathrm{n}}\right)$ such that

$$
\mathrm{E}_{\mathrm{i}}(\overline{\mathrm{x}}) \geq \mathrm{E}_{\mathrm{i}}\left(\mathrm{x}_{\mathrm{d}(\mathrm{i})}, \overline{\mathrm{x}}_{\mathrm{N}-\mathrm{d}(\mathrm{i})}\right) \quad \forall \mathrm{i} \forall \mathrm{x}_{\mathrm{d}(\mathrm{i})} .
$$

We remind the following result.

Preposition: $\overline{\mathrm{x}}$ is an $E$-point if and only if

$$
\begin{array}{cc}
\lambda_{\mathrm{i}}-\mathrm{E}_{\mathrm{i}}\left(\sigma_{\mathrm{d}(\mathrm{i})}, \overline{\mathrm{x}}_{\mathrm{N}-\mathrm{d}(\mathrm{i})}\right)=0 & \forall \sigma_{\mathrm{d}(\mathrm{i})} \in \prod_{\mathrm{j} \in \mathrm{d}(\mathrm{i})} \mathrm{S}\left(\overline{\mathrm{x}}_{\mathrm{j}}\right) \\
\lambda_{\mathrm{i}}-\mathrm{E}_{\mathrm{i}}\left(\sigma_{\mathrm{d}(\mathrm{i})}, \overline{\mathrm{x}}_{\mathrm{N}-\mathrm{d}(\mathrm{i})}\right) \geq 0 & \forall \sigma_{\mathrm{d}(\mathrm{i})} \notin \prod_{\mathrm{j} \in \mathrm{d}(\mathrm{i})} \mathrm{S}\left(\overline{\mathrm{x}}_{\mathrm{j}}\right) \\
\sum_{\sigma_{\mathrm{i}} \in \Sigma_{\mathrm{i}}} \overline{\mathrm{x}}_{\mathrm{i}}\left(\sigma_{\mathrm{i}}\right)=1 & \forall \mathrm{i} \\
\overline{\mathrm{x}}_{\mathrm{i}}\left(\sigma_{\mathrm{i}}\right) \geq 0 \quad \forall \mathrm{i} \quad \forall \sigma_{\mathrm{i}} \in \Sigma_{\mathrm{i}} .
\end{array}
$$

where $S\left(\bar{x}_{j}\right)$ denotes the support of the mixed strategy $\bar{x}_{j}$.

From now on we consider $n$-person games in normal form where all the players have the same cardinality for their pure strategy set: $\mathrm{m}=\left|\Sigma_{\mathrm{i}}\right|$.

In the next paragraph we will study a 7-player diagonal game and a 10-player game with similar d(i) structure. Next we generalize them in a general way. In the third section we study an 8-player game of the game type for the sets d(i) and finally we generalize it to $3 \mathrm{k}+2$-player with similar structure function.

\section{7 and 10-players diagonal games}

Here in this paragraph we will compute explicitly the $E$-points for two games, namely are with 7-players and the other are with 10-players. 
The first one is given with the structure function $d(i)$ as $d(1)=\{1,2,6,7\}, d(2)$ $=\{2,3,7,1\}, d(3)=\{1,2,3,4\}, d(4)=\{2,3,4,5\}, d(5)=\{3,4,5,6\}, d(6)=\{4,5,6$, $7\}, d(7)=\{5,6,7,1\}$. Therefore due to the Preposition if the game is diagonal that is to say

$$
\mathrm{A}_{\mathrm{i}}\left(\sigma_{1} \ldots \sigma_{2}\right)=\mathrm{a}_{\mathrm{i}}\left(\sigma_{\mathrm{i}}\right) \delta\left(\sigma_{\mathrm{i}}, \sigma_{\mathrm{i}+2}, \sigma_{\mathrm{i}+3}, \sigma_{\mathrm{i}+4}\right) \quad \bmod 7 \quad \mathrm{a}_{\mathrm{i}}\left(\sigma_{\mathrm{i}}\right)>0
$$

where

$$
\delta\left(\sigma_{\mathrm{j}_{1}}, \sigma_{\mathrm{j}_{2}}, \sigma_{\mathrm{j}_{3}}, \ldots, \sigma_{\mathrm{j}_{\mathrm{k}}}\right)=\delta\left(\sigma_{\mathrm{j}_{1}}, \sigma_{\mathrm{j}_{2}}\right) \cdots \delta\left(\sigma_{\mathrm{j}_{\mathrm{k}-1}}, \sigma_{\mathrm{j}_{\mathrm{k}}}\right)
$$

where the $\delta$ 's are Krorecker's deltas.

We remind that a completely mixed strategy is a vector $\mathrm{x}=\left(\mathrm{x}_{1}, \ldots, \mathrm{x}_{\mathrm{n}}\right)$ such that $\forall \mathrm{i} \forall \sigma_{\mathrm{i}} \in \sum_{\mathrm{i}} \mathrm{x}_{\mathrm{i}}\left(\sigma_{\mathrm{i}}\right)>0$.

Then is we want to compute the completely mixed E-points for our 7-person game for the Preposition we have to solve

$$
\begin{array}{ll}
\lambda_{1}-\mathrm{a}_{1}(\sigma) \mathrm{x}_{3}(\sigma) \mathrm{x}_{4}(\sigma) \mathrm{x}_{5}(\sigma)=0 & \forall \sigma \\
\lambda_{2}-\mathrm{a}_{2}(\sigma) \mathrm{x}_{4}(\sigma) \mathrm{x}_{5}(\sigma) \mathrm{x}_{6}(\sigma)=0 & \forall \sigma \\
\lambda_{3}-\mathrm{a}_{3}(\sigma) \mathrm{x}_{5}(\sigma) \mathrm{x}_{6}(\sigma) \mathrm{x}_{7}(\sigma)=0 & \forall \sigma \\
\lambda_{4}-\mathrm{a}_{4}(\sigma) \mathrm{x}_{6}(\sigma) \mathrm{x}_{7}(\sigma) \mathrm{x}_{1}(\sigma)=0 & \forall \sigma \\
\lambda_{5}-\mathrm{a}_{5}(\sigma) \mathrm{x}_{2}(\sigma) \mathrm{x}_{1}(\sigma) \mathrm{x}_{2}(\sigma)=0 & \forall \sigma \\
\lambda_{6}-\mathrm{a}_{6}(\sigma) \mathrm{x}_{1}(\sigma) \mathrm{x}_{2}(\sigma) \mathrm{x}_{3}(\sigma)=0 & \forall \sigma \\
\lambda_{7}-\mathrm{a}_{7}(\sigma) \mathrm{x}_{2}(\sigma) \mathrm{x}_{3}(\sigma) \mathrm{x}_{4}(\sigma)=0 & \forall \sigma
\end{array}
$$

For simplicity reasons until we need it we drop the explicit notation of the independent variable that it is to say we write $\mathrm{a}_{\mathrm{i}}=\mathrm{a}_{\mathrm{i}}(\sigma)$ and $\mathrm{x}_{\mathrm{i}}(\sigma)=\mathrm{x}_{\mathrm{i}}$. From (1) calling $\mu_{\mathrm{i}}=\mu_{\mathrm{i}}(\sigma)=\lambda_{\mathrm{i}} / \mathrm{a}_{\mathrm{i}}$ we obtain

$$
\begin{gathered}
x_{6}=\frac{\mu_{2}}{\mu_{1}} x_{3}, x_{7}=\frac{\mu_{3}}{\mu_{2}} x_{4}, x_{1}=\frac{\mu_{4}}{\mu_{3}} x_{5}, x_{2}=\frac{\mu_{5}}{\mu_{4}} x_{6}, x_{3}=\frac{\mu_{6}}{\mu_{5}} x_{7} \\
x_{4}=\frac{\mu_{6}}{\mu_{6}} x_{1}, x_{5}=\frac{\mu_{1}}{\mu_{7}} x_{2} .
\end{gathered}
$$

By replacing terms we have from these last equalities

$$
\mathrm{x}_{2}=\frac{\mu_{3} \mu_{7}}{\mu_{1} \mu_{4}} \mathrm{x}_{1}, \mathrm{x}_{7}=\frac{\mu_{3} \mu_{7}}{\mu_{2} \mu_{6}} \mathrm{x}_{1} .
$$

Replacing them in the fifth equation of (1) we get 


$$
\mu_{5}-x_{1}^{3} \frac{\mu_{3}^{2} \mu_{7}^{2}}{\mu_{2} \mu_{6} \mu_{4} \mu_{1}}=0
$$

In a similar way it is possible to obtain the order equations, or by symmetry arguments, which happen to be

$$
\begin{aligned}
& \mu_{6}-x_{2}^{3} \frac{\mu_{1}^{2} \mu_{4}^{2}}{\mu_{3} \mu_{7} \mu_{5} \mu_{2}}=0 \\
& \mu_{7}-x_{3}^{3} \frac{\mu_{5}^{2} \mu_{2}^{2}}{\mu_{1} \mu_{3} \mu_{4} \mu_{6}}=0
\end{aligned}
$$

From (2) one obtains

$$
\mathrm{x}_{1}^{3}(\sigma)=\frac{\lambda_{1} \lambda_{2} \lambda_{4} \lambda_{5} \lambda_{6}}{\lambda_{3}^{2} \lambda_{7}^{2}} \frac{\mathrm{a}_{3}^{2}(\sigma) \mathrm{a}_{7}^{2}(\sigma)}{\mathrm{a}_{1}(\sigma) \mathrm{a}_{2}(\sigma) \mathrm{a}_{4}(\sigma) \mathrm{a}_{5}(\sigma) \mathrm{a}_{6}(\sigma)}
$$

Using the condition $\sum_{\sigma} \mathrm{x}_{1}(\sigma)=1$ one gets

$$
\mathrm{b}_{1}=\left(\frac{1}{\sum_{\sigma}\left(\frac{\mathrm{a}_{3}^{2}(\sigma) \mathrm{a}_{7}^{2}(\sigma)}{\mathrm{a}_{1}(\sigma) \mathrm{a}_{2}(\sigma) \mathrm{a}_{4}(\sigma) \mathrm{a}_{5}(\sigma) \mathrm{a}_{6}(\sigma)}\right)^{1 / 3}}\right)^{3}=\frac{\lambda_{1} \lambda_{2} \lambda_{4} \lambda_{5} \lambda_{6}}{\lambda_{3}^{2} \lambda_{7}^{2}}
$$

Similarly for the other two equations in (3)

$$
\begin{aligned}
\mathrm{x}_{2}^{3}(\sigma) & =\frac{\lambda_{2} \lambda_{3} \lambda_{5} \lambda_{6} \lambda_{7}}{\lambda_{1}^{2} \lambda_{4}^{2}} \frac{\mathrm{a}_{1}^{2}(\sigma) \mathrm{a}_{4}^{2}(\sigma)}{\mathrm{a}_{2}(\sigma) \mathrm{a}_{3}(\sigma) \mathrm{a}_{5}(\sigma) \mathrm{a}_{6}(\sigma) \mathrm{a}_{7}(\sigma)} \\
\mathrm{x}_{3}^{3}(\sigma) & =\frac{\lambda_{1} \lambda_{3} \lambda_{4} \lambda_{6} \lambda_{7}}{\lambda_{2}^{2} \lambda_{5}^{2}} \frac{\mathrm{a}_{2}^{2}(\sigma) \mathrm{a}_{5}^{2}(\sigma)}{\mathrm{a}_{1}(\sigma) \mathrm{a}_{3}(\sigma) \mathrm{a}_{4}(\sigma) \mathrm{a}_{6}(\sigma) \mathrm{a}_{7}(\sigma)}
\end{aligned}
$$

and then

$$
\mathrm{b}_{2}=\left(\frac{1}{\sum_{\sigma}\left(\frac{\mathrm{a}_{1}^{2}(\sigma) \mathrm{a}_{4}^{2}(\sigma)}{\mathrm{a}_{2}(\sigma) \mathrm{a}_{3}(\sigma) \mathrm{a}_{5}(\sigma) \mathrm{a}_{6}(\sigma) \mathrm{a}_{7}(\sigma)}\right)^{1 / 3}}\right)^{3}=\frac{\lambda_{2} \lambda_{3} \lambda_{5} \lambda_{6} \lambda_{7}}{\lambda_{1}^{2} \lambda_{4}^{2}}
$$




$$
\mathrm{b}_{3}=\left(\frac{1}{\sum_{\sigma}\left(\frac{\mathrm{a}_{2}^{2}(\sigma) \mathrm{a}_{5}^{2}(\sigma)}{\mathrm{a}_{1}(\sigma) \mathrm{a}_{3}(\sigma) \mathrm{a}_{4}(\sigma) \mathrm{a}_{6}(\sigma) \mathrm{a}_{7}(\sigma)}\right)^{1 / 3}}\right)^{3}=\frac{\lambda_{1} \lambda_{3} \lambda_{4} \lambda_{6} \lambda_{7}}{\lambda_{2}^{2} \lambda_{5}^{2}}
$$

Now multiplying these three last numbers we get

$$
\lambda_{6}^{3}=b_{1} b_{2} b_{3}
$$

Thus the value

$$
\lambda_{6}=\left(b_{1} b_{2} b_{3}\right)^{1 / 3}
$$

is determined. By analogous arguments it is possible to derive

$$
\begin{gathered}
\lambda_{7}=\left(b_{2} b_{3} b_{4}\right)^{1 / 3}, \lambda_{1}=\left(b_{3} b_{4} b_{5}\right)^{1 / 3}, \lambda_{2}=\left(b_{4} b_{5} b_{6}\right)^{1 / 3}, \lambda_{3}=\left(b_{5} b_{6} b_{7}\right)^{1 / 3}, \\
\lambda_{4}=\left(b_{6} b_{7} b_{1}\right)^{1 / 3}, \lambda_{5}=\left(b_{7} b_{1} b_{2}\right)^{1 / 3}
\end{gathered}
$$

where the value $b_{i}$ are obtained by symmetry from the previous $b_{1}, b_{2}$ and $b_{3}$. Thus the problem of getting an $E$-point completely mixed for this game is solved. It is clear that it is the unique $E$-point completely mixed that this game possesses.

Now we are going to study a 10-person game. Consider the game with structure function $\mathrm{d}(\mathrm{i})=\{\mathrm{i}, \mathrm{i}+1, \mathrm{i}+5, \mathrm{i}+6, \ldots, 10,1,2, \ldots, \mathrm{i}-1\} \bmod 10$ and payoff function

$$
\mathrm{A}_{\mathrm{i}}\left(\sigma_{1}, \ldots, \sigma_{10}\right)=\mathrm{a}_{\mathrm{i}}\left(\sigma_{\mathrm{i}}\right) \delta\left(\sigma_{\mathrm{i}}, \sigma_{\mathrm{i}+2}, \sigma_{\mathrm{i}+3}, \sigma_{\mathrm{i}+4}\right) \quad \mathrm{a}_{\mathrm{i}}\left(\sigma_{\mathrm{i}}\right)>0
$$

Then from equation (1), the E-point has to satisfy

$$
\mu_{\mathrm{i}}-\mathrm{x}_{\mathrm{i}+2} \mathrm{x}_{\mathrm{i}+3} \mathrm{x}_{\mathrm{i}+4}=0 \quad \bmod 10
$$

From here we get by dividing two consecutive

$$
\mathrm{x}_{\mathrm{i}+5}=\frac{\mu_{\mathrm{i}+1}}{\mu_{\mathrm{i}}} \mathrm{x}_{\mathrm{i}+2} \quad \bmod 10
$$

and by replacing the respective x's we get

$$
\begin{gathered}
x_{6}=\frac{\mu_{2}}{\mu_{1}} x_{3}, x_{7}=\frac{\mu_{3}}{\mu_{2}} x_{4}, x_{8}=\frac{\mu_{4}}{\mu_{3}} x_{5}, x_{9}=\frac{\mu_{3} \mu_{10}}{\mu_{4} \mu_{1}} x_{8} \\
x_{10}=\frac{\mu_{3} \mu_{10} \mu_{7}}{\mu_{5} \mu_{2} \mu_{9}} x_{8}, x_{1}=\frac{\mu_{7}}{\mu_{6}} x_{8}, x_{3}=\frac{\mu_{9}}{\mu_{8}} x_{10}, x_{4}=\frac{\mu_{10} \mu_{7}}{\mu_{6} \mu_{9}} x_{8}, x_{5}=\frac{\mu_{1}}{\mu_{10}} x_{2}
\end{gathered}
$$

which we wish to have them in term of $x_{8}$. Replacing in (4) with $i=6$ we obtain 


$$
\mathrm{x}_{8}^{3}=\frac{\mu_{6} \mu_{9} \mu_{2} \mu_{5} \mu_{8} \mu_{1} \mu_{4}}{\mu_{3}^{2} \mu_{7}^{2} \mu_{10}^{2}}
$$

Doing the same thing with respect to $\mathrm{x}_{9}$ and $\mathrm{x}_{10}$ and respectively using equation (4) with $i=7$, and 8 we get

$$
\mathrm{x}_{9}^{3}=\frac{\mu_{7} \mu_{10} \mu_{3} \mu_{6} \mu_{9} \mu_{2} \mu_{5}}{\mu_{3}^{2} \mu_{7}^{2} \mu_{10}^{2}}
$$

and

$$
x_{10}^{3}=\frac{\mu_{8} \mu_{1} \mu_{4} \mu_{2} \mu_{10} \mu_{3} \mu_{6}}{\mu_{5}^{2} \mu_{9}^{2} \mu_{2}^{2}}
$$

From (3) are gets, using $\sum_{\sigma} x_{8}(\sigma)=1$ that

$$
\mathrm{b}_{8}=\left(\frac{1}{\sum_{\sigma}\left(\frac{\mathrm{a}_{3}^{2}(\sigma) \mathrm{a}_{7}^{2}(\sigma) \mathrm{a}_{10}^{2}(\sigma)}{\mathrm{a}_{6}(\sigma) \mathrm{a}_{9}(\sigma) \mathrm{a}_{2}(\sigma) \mathrm{a}_{5}(\sigma) \mathrm{a}_{8}(\sigma) \mathrm{a}_{1}(\sigma) \mathrm{a}_{4}(\sigma)}\right)^{1 / 3}}\right)^{3}=\frac{\lambda_{6} \lambda_{9} \lambda_{2} \lambda_{5} \lambda_{8} \lambda_{1} \lambda_{10}}{\lambda_{3}^{2} \lambda_{7}^{2} \lambda_{10}^{2}}
$$

In an analogous way from (6) we get

$$
\begin{gathered}
\mathrm{b}_{9}=\left(\frac{1}{\sum_{\sigma}\left(\frac{\mathrm{a}_{1}^{2}(\sigma) \mathrm{a}_{4}^{2}(\sigma) \mathrm{a}_{8}^{2}(\sigma)}{\mathrm{a}_{7}(\sigma) \mathrm{a}_{10}(\sigma) \mathrm{a}_{3}(\sigma) \mathrm{a}_{6}(\sigma) \mathrm{a}_{9}(\sigma) \mathrm{a}_{2}(\sigma) \mathrm{a}_{5}(\sigma)}\right)^{1 / 3}}\right)^{3}=\frac{\lambda_{7} \lambda_{10} \lambda_{3} \lambda_{6} \lambda_{9} \lambda_{2} \lambda_{10}}{\lambda_{1}^{2} \lambda_{4}^{2} \lambda_{8}^{2}} \\
\mathrm{~b}_{10}=\left(\frac{1}{\sum_{\sigma}\left(\frac{\mathrm{a}_{5}^{2}(\sigma) \mathrm{a}_{9}^{2}(\sigma) \mathrm{a}_{2}^{2}(\sigma)}{\mathrm{a}_{8}(\sigma) \mathrm{a}_{1}(\sigma) \mathrm{a}_{4}(\sigma) \mathrm{a}_{7}(\sigma) \mathrm{a}_{10}(\sigma) \mathrm{a}_{3}(\sigma) \mathrm{a}_{6}(\sigma)}\right)^{3}}\right)^{3 / 3}=\frac{\lambda_{8} \lambda_{1} \lambda_{4} \lambda_{2} \lambda_{10} \lambda_{3} \lambda_{6}}{\lambda_{5}^{2} \lambda_{9}^{2} \lambda_{2}^{2}}
\end{gathered}
$$

Multiplying the last three b's we get

$$
\lambda_{6}^{3}=b_{8} b_{9} b_{19}
$$

Thus $\lambda_{6}$ is completely determined. Doing the same thing all the others $\lambda^{\prime} \mathrm{s}$ might be determined. Replacing these values in the corresponding expressions of the x's the unique completely mixed $E$-points can be obtained. 
As a remark we would like to mention that any point $(\bar{\sigma}, \bar{\sigma}, \bar{\sigma}, \ldots, \bar{\sigma})$ is an $E$ point. Then the both previous games have exactly

$$
\sum_{i=1}^{m}\left(\begin{array}{c}
m \\
i
\end{array}\right)=2^{m}-1
$$

$E$-points. Only one of them is completely mixed.

\section{A general study for a $3 k+1$ person game with $k \geq 1$}

Now in this section we will study and compute the E-points in a general diagonal game with $3 \mathrm{k}+1$ with $\mathrm{k} \geq 1$ players. The structure function is given by $d(i)=\{i, i+1, i+2, i+5, i+6, \ldots, 3 k+1,1,2, \ldots, i-1\}$ with payoff functions given by

$$
\mathrm{A}_{\mathrm{i}}\left(\sigma_{\mathrm{i}}, \ldots, \sigma_{3 \mathrm{k}+1}\right)=\mathrm{a}_{\mathrm{i}}\left(\sigma_{\mathrm{i}}\right) \delta\left(\sigma_{\mathrm{i}}, \sigma_{\mathrm{i}+2}, \sigma_{\mathrm{i}+3}, \sigma_{\mathrm{i}+4}\right) \quad \bmod 3 \mathrm{k}+1
$$

Therefore the equation (1) in this case is

$$
\bar{\mu}_{i}-x_{i+2} x_{i+3} x_{i+4}=0 \quad \bmod 3 k+1 .
$$

or

$$
\mu_{i+2}-x_{i+2} x_{i+3} x_{i+4}=0 \quad \bmod 3 k+1
$$

where $\bar{\mu}_{\mathrm{i}}=\mu_{\mathrm{i}+2}$.

From two consecutive two equations of (8) we get

$$
x_{i+3}=\frac{\mu_{i+1}}{\mu_{i}} x_{i}=S_{i+1} x_{i}
$$

and replacing this

$$
\mathrm{x}_{\mathrm{i}+3}=\prod_{\mathrm{s}=1}^{\mathrm{r}} \mathrm{S}_{\mathrm{i}+1-3 \mathrm{~s}} \mathrm{x}_{\mathrm{i}-3 \mathrm{r}} \quad \bmod 3 \mathrm{k}+1
$$

Now let us permit to arrange the numbers in strips in a natural way

$$
\begin{aligned}
& 14 \ldots 3 \mathrm{k}-2 \quad \underline{3 \mathrm{k}+1} \quad 3 \mathrm{k}+4 \equiv 3 \ldots \ldots 6 \mathrm{k}+1 \quad 6 \mathrm{k}+4 \equiv 2 \ldots \\
& 25 \ldots 3 \mathrm{k}-1 \quad 3 \mathrm{k}+2 \equiv 1 \quad \ldots \ldots .6 \mathrm{k}+2 \equiv 3 \mathrm{k}+1 \quad 6 \mathrm{k}+5 \equiv 3 \ldots \\
& 35 \ldots 3 \mathrm{k} \quad 3 \mathrm{k}+3 \equiv 3 \quad \ldots \ldots 6 \mathrm{k}+3 \equiv 1 \\
& \begin{array}{ll}
\ldots \ldots & 9 \mathrm{k}+4 \equiv 1 \\
\ldots \ldots & 9 \mathrm{k}+5 \equiv 2
\end{array} \\
& \text {.... } \underline{9 \mathrm{k}+3} \equiv \mathrm{k}+1 \quad 9 \mathrm{k}+6 \equiv 3 .
\end{aligned}
$$


Take $\mathrm{p} \leq \mathrm{k}$ and consider the $3 \mathrm{p}+1$-expression

$$
\mu_{3 p+1}-x_{3 p+1} x_{3 p+2} x_{3 p+3}=0
$$

Next we are going to express $x_{3 p+2}$ and $x_{3 p+3}$ in terms of $x_{3 p+1}$. For this take $3 p+3+3 k+1=3(p+k)+4=i+3$ or $i=3(p+k)+1$ in (9) with $r=k$. Then we have

$$
\mathrm{x}_{3 \mathrm{p}+3}=\prod_{\mathrm{s}=0}^{\mathrm{k}} \mathrm{S}_{3(\mathrm{p}+\mathrm{k})+2-3 \mathrm{~s}} \mathrm{x}_{3 \mathrm{p}+1}
$$

Now taking $v=3(p+2 k)+1$ and $r=2 k$ replacing in (9) one gets

$$
\mathrm{x}_{3 \mathrm{p}+2}=\prod_{\mathrm{s}=0}^{2 \mathrm{k}} \mathrm{S}_{3(\mathrm{p}+2 \mathrm{k})+2-3 \mathrm{~s}} \mathrm{x}_{3 \mathrm{p}+1}
$$

and replacing these values in (11) it is derived

$$
\mu_{3 p+1}-x_{3 p+1}^{3} \prod_{s=0}^{k} S_{3(p+k)+2-3 s} \prod_{s=0}^{2 k} S_{3(p+2 k)+2-3 s}=0
$$

Now consider the number $3 p+2$ with $p \leq k$. Then for it we have

$$
\mu_{3 p+2}-x_{3 p+2} x_{3 p+3} x_{3 p+4}=0
$$

Putting in (9) $i=3(p+k)+2$ and $r=k$ we get

$$
\mathrm{x}_{3 \mathrm{p}+4}=\prod_{\mathrm{k}=0}^{\mathrm{k}} \mathrm{S}_{3(\mathrm{p}+\mathrm{k})+3-3 \mathrm{~s}} \mathrm{x}_{3 \mathrm{p}+2}
$$

and with $\mathrm{i}=3(\mathrm{p}+2 \mathrm{k})+2$ and $\mathrm{r}=2 \mathrm{k}$

$$
\mathrm{x}_{3 \mathrm{p}+3}=\prod_{\mathrm{k}=0}^{2 \mathrm{k}} \mathrm{S}_{3(\mathrm{p}+2 \mathrm{k})+3-3 \mathrm{~s}} \mathrm{x}_{3 \mathrm{p}+2}
$$

which replaced in (14) provides

$$
\mu_{3 p+2}-x_{3 p+2}^{3} \prod_{s=0}^{k} S_{3(p+k)+3-3 s} \prod_{s=0}^{2 k} S_{3(p+2 k)+3-3 s}=0
$$

Finally with $i=3(p+k)+3$ and $r=k$ an the one hand and $i=3(p+2 k)+3$ and an the other using (9) and the replacing the corresponding values of $\mathrm{x}_{3 \mathrm{p}+4}$ and $\mathrm{x}_{3 \mathrm{p}+5}$ it is easy to derive

$$
\mu_{3 p+3}-x_{3 p+3}^{3} \prod_{k=0}^{k} S_{3(p+k)+4-3 s} \prod_{s=0}^{2 k} S_{3(p+2 k)+3-3 s}=0
$$


Now replacing the values of $S_{i}$ in $\left(12^{\prime}\right)$ and using the condition $\sum_{\sigma} x_{3 p+1}(\sigma)=1$ it holds

$$
\begin{gathered}
\lambda_{3 \mathrm{p}-1} \frac{\prod_{\mathrm{s}=0}^{\mathrm{k}} \lambda_{3(\mathrm{p}+\mathrm{k})-1-3 \mathrm{~s}}}{\prod_{\mathrm{s}=0}^{\mathrm{k}} \lambda_{3(\mathrm{p}+\mathrm{k})-3 \mathrm{~s}}} \frac{\prod_{\mathrm{s}=0}^{2 \mathrm{k}} \lambda_{3(\mathrm{p}+2 \mathrm{k})-1-3 \mathrm{~s}}}{\prod_{\mathrm{s}=0}^{2 \mathrm{k}} \lambda_{3(\mathrm{p}+2 \mathrm{k})-3 \mathrm{~s}}}=\mathrm{b}_{3 \mathrm{p}+1}= \\
=\left(\frac{1}{\sum_{\sigma}\left(\frac{1}{\mathrm{a}_{3 \mathrm{p}-1}(\sigma)} \frac{\prod_{\mathrm{s}=0}^{\mathrm{k}} \mathrm{a}_{3(\mathrm{p}+\mathrm{k})-3 \mathrm{~s}}(\sigma)}{\prod_{\mathrm{s}=0}^{2 \mathrm{k}} \mathrm{a}_{3(\mathrm{p}+\mathrm{k})-1-3 \mathrm{~s}}(\sigma)} \frac{\prod_{\mathrm{s}=0}^{2 \mathrm{k}} \mathrm{a}_{3(\mathrm{p}+2 \mathrm{k})-2 \mathrm{~s}}(\sigma)}{\prod_{\mathrm{s}=0}^{2 \mathrm{k}} \mathrm{a}_{3(\mathrm{p}+2 \mathrm{k})-1-3 \mathrm{~s}}(\sigma)}\right)^{1 / 3}}\right)^{3}
\end{gathered}
$$

Doing the same thing with (17), one derives

$$
\begin{gathered}
\lambda_{3 \mathrm{p}} \frac{\prod_{\mathrm{s}=0}^{\mathrm{k}} \lambda_{3(\mathrm{p}+\mathrm{k})-3 \mathrm{~s}}}{\prod_{\mathrm{s}=0}^{\mathrm{k}} \lambda_{3(\mathrm{p}+\mathrm{k})+1-3 \mathrm{~s}}} \frac{\prod_{\mathrm{s}=0}^{2 \mathrm{k}} \lambda_{3(\mathrm{p}+2 \mathrm{k})-3 \mathrm{~s}}}{\prod_{\mathrm{s}=0}^{2 \mathrm{k}} \lambda_{3(\mathrm{p}+2 \mathrm{k})+1-3 \mathrm{~s}}}=\mathrm{b}_{3 \mathrm{p}+2}= \\
=\left(\frac{1}{\sum_{\sigma}\left(\frac{1}{\mathrm{a}_{3 \mathrm{p}}(\sigma)} \frac{\prod_{\mathrm{s}=0}^{\mathrm{k}} \mathrm{a}_{3(\mathrm{p}+\mathrm{k})+1-3 \mathrm{~s}}(\sigma)}{\prod_{\mathrm{s}=0}^{\mathrm{k}} \mathrm{a}_{3(\mathrm{p}+\mathrm{k})-3 \mathrm{~s}}(\sigma)} \frac{\prod_{\mathrm{s}=0}^{2 \mathrm{k}} \mathrm{a}_{3(\mathrm{p}+2 \mathrm{k})+1-3 \mathrm{~s}}(\sigma)}{\prod_{\mathrm{s}=0}^{2 \mathrm{k}} \mathrm{a}_{3(\mathrm{p}+2 \mathrm{k})-3 \mathrm{~s}}(\sigma)}\right)^{1 / 3}}\right)^{3}
\end{gathered}
$$

Finally using (18) it follows

$$
\begin{aligned}
& \lambda_{3 \mathrm{p}+1} \frac{\prod_{\mathrm{s}=0}^{\mathrm{k}} \lambda_{3(\mathrm{p}+\mathrm{k})+1-3 \mathrm{~s}}}{\prod_{\mathrm{s}=0}^{\mathrm{k}} \lambda_{3(\mathrm{p}+\mathrm{k})+2-3 \mathrm{~s}}} \frac{\prod_{\mathrm{s}=0}^{2 \mathrm{k}} \lambda_{3(\mathrm{p}+2 \mathrm{k})+1-3 \mathrm{~s}}}{\prod_{\mathrm{s}=0}^{2 \mathrm{k}} \lambda_{3(\mathrm{p}+2 \mathrm{k})+2-3 \mathrm{~s}}}=\mathrm{b}_{3 \mathrm{p}+1}= \\
& =\left(\frac{1}{\sum_{\sigma}\left(\frac{1}{a_{3 p+1}(\sigma)} \frac{\prod_{s=0}^{\mathrm{k}} \mathrm{a}_{3(\mathrm{p}+\mathrm{k})+2-3 \mathrm{~s}}(\sigma)}{\prod_{\mathrm{s}=0}^{\mathrm{k}} \mathrm{a}_{3(\mathrm{p}+\mathrm{k})+1-1-3 \mathrm{~s}}(\sigma)} \frac{\prod_{\mathrm{s}=0}^{2 \mathrm{k}} \mathrm{a}_{3(\mathrm{p}+2 \mathrm{k})+2-3 \mathrm{~s}}(\sigma)}{\prod_{\mathrm{s}=0}^{2 \mathrm{k}} \mathrm{a}_{3(\mathrm{p}+2 \mathrm{k})+1-3 \mathrm{~s}}(\sigma)}\right)^{1 / 3}}\right)^{3}
\end{aligned}
$$

Now multiplying the equalities (19), (20) and (21) it holds to

$$
\mathrm{b}_{3 \mathrm{p}+1} \mathrm{~b}_{3 \mathrm{p}+2} \mathrm{~b}_{3 \mathrm{p}+3}=\frac{\lambda_{3 \mathrm{p}-1}^{3} \lambda_{3 \mathrm{p}} \lambda_{3 \mathrm{p}+1}}{\lambda_{3(\mathrm{p}+\mathrm{k})+2} \lambda_{3(\mathrm{p}+2 \mathrm{k})+2}}
$$


but

$$
3 p+1+3 k+1=3(p+k)+2 \equiv 3 p+1
$$

and

$$
3 p+2(3 k+1) \equiv 3(p+2 k)+2 \quad \bmod 3 k+1
$$

then

$$
\lambda_{3 p-1}=\left(b_{3 p+1} b_{3 p+2} b_{3 p+3}\right)^{1 / 3} .
$$

In a similar way or by symmetry it is possible to derive

$$
\lambda_{3 p}=\left(b_{3 p+2} b_{3 p+3} b_{3 p+4}\right)^{1 / 3}
$$

and

$$
\lambda_{3 \mathrm{p}+1}=\left(\mathrm{b}_{3 \mathrm{p}+3} \mathrm{~b}_{3 \mathrm{p}+4} \mathrm{~b}_{3 \mathrm{p}+5}\right)^{1 / 3}
$$

and in this way we have obtained the explicit value of the $\lambda$ 's and replacing them in the corresponding place of (13), (14) or (18) one gets the corresponding value of the x's. Thus the problem is solved positively.

We remind as in [4] that the point $(\bar{\sigma}, \bar{\sigma}, \ldots, \bar{\sigma})$ is an $E$-point. Therefore there are exactly

$$
\sum_{\mathrm{i}=1}^{\mathrm{m}}\left(\begin{array}{c}
\mathrm{m} \\
\mathrm{i}
\end{array}\right)=2^{\mathrm{m}}-1
$$

E-points which might be computed accordingly. Any way there is only one E-point completely mixed. The phenomenon appears in all the games studied here and for this reason we do not repeat the same argument every time.

\section{A general study for a $3 k+2$ person game with $k \geq 1$}

In this section we are going to study the same game given in the previous paragraph but when the numbers of players are $3 k+2$ with $k \geq 1$. Before to study the general case with an arbitrary $\mathrm{k}$ consider the case of $\mathrm{k}=2$. That is to say 8 . Therefore the corresponding equation become

$$
\lambda_{\mathrm{i}}-\mathrm{a}_{\mathrm{i}} \mathrm{x}_{\mathrm{i}+2} \mathrm{x}_{\mathrm{i}+3} \mathrm{x}_{\mathrm{i}+4}=0 \quad \bmod 8
$$

and from here 


$$
\begin{gathered}
x_{6}=\frac{\mu_{2}}{\mu_{1}} x_{3}, x_{7}=\frac{\mu_{3}}{\mu_{2}} x_{4}, x_{8}=\frac{\mu_{4}}{\mu_{3}} x_{5}=\frac{\mu_{4} \mu_{1} \mu_{6}}{\mu_{3} \mu_{8} \mu_{5}} x_{7}, x_{1}=\frac{\mu_{5} \mu_{2} \mu_{7}}{\mu_{4} \mu_{1} \mu_{8}} x_{8} \\
x_{2}=\frac{\mu_{6}}{\mu_{5}} x_{7}=\frac{\mu_{6} \mu_{3} \mu_{8}}{\mu_{4} \mu_{1} \mu_{6}} x_{8}, x_{3}=\frac{\mu_{7}}{\mu_{6}} x_{8}, x_{4}=\frac{\mu_{8}}{\mu_{7}} x_{1}, x_{5}=\frac{\mu_{1}}{\mu_{8}} x_{2} .
\end{gathered}
$$

Thus replacing in (24) with $i=5$

$$
x_{7}^{3}=\frac{\mu_{3}^{2} \mu_{8}^{2} \mu_{5}^{2}}{\mu_{1} \mu_{2} \mu_{4} \mu_{6} \mu_{7}}
$$

By symmetry

$$
\begin{aligned}
& x_{8}^{3}=\frac{\mu_{6}^{2} \mu_{4}^{2} \mu_{1}^{2}}{\mu_{2} \mu_{5} \mu_{7} \mu_{8} \mu_{3}} \\
& x_{1}^{3}=\frac{\mu_{7}^{2} \mu_{2}^{2} \mu_{5}^{2}}{\mu_{3} \mu_{6} \mu_{8} \mu_{1} \mu_{4}} \\
& x_{2}^{3}=\frac{\mu_{8}^{2} \mu_{3}^{2} \mu_{6}^{2}}{\mu_{4} \mu_{7} \mu_{1} \mu_{2} \mu_{5}} \\
& x_{3}^{3}=\frac{\mu_{1}^{2} \mu_{4}^{2} \mu_{7}^{2}}{\mu_{5} \mu_{8} \mu_{2} \mu_{3} \mu_{6}} \\
& x_{4}^{3}=\frac{\mu_{2}^{2} \mu_{5}^{2} \mu_{8}^{2}}{\mu_{6} \mu_{1} \mu_{3} \mu_{4} \mu_{7}} \\
& x_{5}^{3}=\frac{\mu_{3}^{2} \mu_{6}^{2} \mu_{1}^{2}}{\mu_{7} \mu_{2} \mu_{4} \mu_{5} \mu_{8}} \\
& x_{6}^{3}=\frac{\mu_{4}^{2} \mu_{7}^{2} \mu_{2}^{2}}{\mu_{8} \mu_{3} \mu_{5} \mu_{6} \mu_{1}}
\end{aligned}
$$

Introducing the corresponding values b's it is possible to obtain

$$
\lambda_{i}^{3}=b_{i+2} b_{i+3} b_{i+4}
$$

This expresses the fact that in the general case with $3 \mathrm{k}+2$ players we should obtain an analogous solution as in the previous general case with $3 \mathrm{k}+1$ players.

Indeed consider the general equation for the general case $3 \mathrm{k}+2$ :

$$
\bar{\mu}_{\mathrm{i}}-\mathrm{x}_{\mathrm{i}+2} \mathrm{x}_{\mathrm{i}+3} \mathrm{x}_{\mathrm{i}+4}=0 \quad \bmod 3 \mathrm{k}+2
$$

where $\bar{\mu}_{\mathrm{i}}=\lambda_{\mathrm{i}} / \mathrm{a}_{\mathrm{i}}, \bar{\mu}_{\mathrm{i}}=\mu_{\mathrm{i}+2}$ :

$$
\mu_{i+2}-x_{i+2} x_{i+3} x_{i+4}=0 \quad \bmod 3 k+2
$$


From this we get the following recursive relations

$$
\mathrm{x}_{\mathrm{i}+3}=\prod_{\mathrm{s}=0}^{\mathrm{r}} \mathrm{S}_{\mathrm{i}+1-3 \mathrm{~s}} \mathrm{x}_{\mathrm{i}-3 \mathrm{~s}} \quad \bmod 3 \mathrm{k}+2
$$

Now we arrange the numbers in strips in a natural way

$$
\begin{array}{lcrr}
3 \mathrm{k}+1 & 3 \mathrm{k}+4 \equiv 2 \ldots \ldots 6 \mathrm{k}+1 & \underline{6 \mathrm{k}+4} \equiv 2 & 6 \mathrm{k}+7 \equiv 2 \ldots \\
\frac{3 \mathrm{k}+2}{3 \mathrm{k}+3 \equiv 1} & 3 \mathrm{k}+5 \equiv 5 \ldots \ldots 6 \mathrm{k}+2 & 6 \mathrm{k}+5 \equiv 1 & \ldots \\
\ldots \ldots 6 \mathrm{k}+3 & 6 \mathrm{k}+6 \equiv 2 & \\
& & \\
\ldots \ldots & 9 \mathrm{k}+7 \equiv 1 \\
\ldots \ldots & 9 \mathrm{k}+8 \equiv 2 \\
& \ldots \ldots \mathrm{gk}+6 \equiv 3 \mathrm{k}+2 & 9 \mathrm{k}+9 \equiv 3 .
\end{array}
$$

Consider in (27) $i=3 p+1$ with $p \leq k$. Then

$$
\mu_{3 \mathrm{p}+1}-\mathrm{x}_{3 \mathrm{p}+1} \mathrm{x}_{3 \mathrm{p}+2} \mathrm{x}_{3 \mathrm{p}+3}=0
$$

Taking $\mathrm{i}=3(\mathrm{p}+\mathrm{k})+1$ and $\mathrm{r}=\mathrm{k}$ in (28) we have

$$
\mathrm{x}_{3 \mathrm{p}+2}=\prod_{\mathrm{s}=0}^{\mathrm{k}} \mathrm{S}_{3(\mathrm{p}+\mathrm{k})+2-3 \mathrm{~s}} \mathrm{x}_{3 \mathrm{p}+1}
$$

On the other hand if $\mathrm{i}=3(\mathrm{p}+2 \mathrm{k})+4$ and $\mathrm{r}=2 \mathrm{k}+1$ then

$$
\mathrm{x}_{3 \mathrm{p}+3}=\prod_{\mathrm{s}=0}^{2 \mathrm{k}+1} \mathrm{~S}_{3(\mathrm{p}+2 \mathrm{k})+5-3 \mathrm{~s}} \mathrm{x}_{3 \mathrm{p}+1}
$$

Replacing these amounts in (29) we obtain

$$
\mu_{3 p+1}-x_{3 p+1}^{3} \prod_{s=0}^{k} S_{3(p+k)+2-3 s} \prod_{s=0}^{2 k+1} S_{3(p+2 k)+5-3 s}=0
$$

In a similar way with $3 p+2$

$$
\mu_{3 p+2}-x_{3 p+2} x_{3 p+3} x_{3 p+4}=0
$$

Taking $\mathrm{i}=3(\mathrm{p}+\mathrm{k})+2$ and $\mathrm{r}=\mathrm{k}$ in (28) we get

$$
\mathrm{x}_{3 \mathrm{p}+3}=\prod_{\mathrm{s}=0}^{\mathrm{k}} \mathrm{S}_{3(\mathrm{p}+\mathrm{k})+3-3 \mathrm{~s}} \mathrm{x}_{3 \mathrm{p}+2}
$$

On the other hand with $\mathrm{i}=3(\mathrm{p}+2 \mathrm{k})+5$ and $\mathrm{r}=2 \mathrm{k}+1$ it follows

$$
\mathrm{x}_{3 \mathrm{p}+4}=\prod_{\mathrm{s}=0}^{2 \mathrm{k}+} \mathrm{S}_{3(\mathrm{p}+2 \mathrm{k})+6-3 \mathrm{~s}} \mathrm{x}_{3 \mathrm{p}+2}
$$

By replacing these last two amounts in (33) it turns out 


$$
\mu_{3 p+2}-x_{3 p+2}^{3} \prod_{s=0}^{k} S_{3(p+k)+3-3 s} \prod_{s=0}^{2 k+1} S_{3(p+2 k)+6-3 s}=0
$$

Finally by symmetry it is easy to get

$$
\mu_{3 \mathrm{p}+3}-\mathrm{x}_{3 \mathrm{p}+3}^{3} \prod_{\mathrm{s}=0}^{\mathrm{k}} \mathrm{S}_{3(\mathrm{p}+\mathrm{k})+4-3 \mathrm{~s}} \prod_{\mathrm{s}=0}^{2 \mathrm{k}+1} \mathrm{~S}_{3(\mathrm{p}+2 \mathrm{k})+7-3 \mathrm{~s}}=0
$$

Using the (32), (36) and (37) we get

$$
\lambda_{3 \mathrm{p}-1} \frac{\prod_{\mathrm{s}=0}^{\mathrm{k}} \lambda_{3(\mathrm{p}+\mathrm{k})-1-3 \mathrm{~s}}}{\prod_{\mathrm{s}=0}^{\mathrm{k}} \lambda_{3(\mathrm{p}+\mathrm{k})-3 \mathrm{~s}}} \frac{\prod_{\mathrm{s}=0}^{2 \mathrm{k}+1} \lambda_{3(\mathrm{p}+2 \mathrm{k})+2-3 \mathrm{~s}}}{\prod_{\mathrm{s}=0}^{2 \mathrm{k}+1} \lambda_{3(\mathrm{p}+2 \mathrm{k})+3-3 \mathrm{~s}}}=\mathrm{b}_{3 \mathrm{p}+1}
$$

$$
\begin{aligned}
& =\left(\frac{1}{\sum_{\sigma}\left(\frac{1}{\mathrm{a}_{3 \mathrm{p}+1}(\sigma)} \frac{\prod_{\mathrm{s}=0}^{\mathrm{k}} \mathrm{a}_{3(\mathrm{p}+\mathrm{k})-3 \mathrm{~s}}(\sigma)}{\prod_{\mathrm{s}=0}^{\mathrm{k}} \mathrm{a}_{3(\mathrm{p}+\mathrm{k})-1-3 \mathrm{~s}}(\sigma)} \frac{\prod_{\mathrm{s}=0}^{2 \mathrm{k}+1} \mathrm{a}_{3(\mathrm{p}+2 \mathrm{k})+3-3 \mathrm{~s}}(\sigma)}{\prod_{\mathrm{s}=0}^{2 \mathrm{k}+1} \mathrm{a}_{3(\mathrm{p}+2 \mathrm{k})+2-3 \mathrm{~s}}(\sigma)}\right)^{1 / 3}}\right)^{3} \\
& \lambda_{3 \mathrm{p}} \frac{\prod_{\mathrm{s}=0}^{\mathrm{k}} \lambda_{3(\mathrm{p}+\mathrm{k})-3 \mathrm{~s}}}{\prod_{\mathrm{s}=0}^{\mathrm{k}} \lambda_{3(\mathrm{p}+\mathrm{k})+1-3 \mathrm{~s}}} \frac{\prod_{\mathrm{s}=0}^{2 \mathrm{k}+1} \lambda_{3(\mathrm{p}+2 \mathrm{k})+3-3 \mathrm{~s}}}{\prod_{\mathrm{s}=0}^{2 \mathrm{k}+1} \lambda_{3(\mathrm{p}+2 \mathrm{k})+4-3 \mathrm{~s}}}=\mathrm{b}_{3 \mathrm{p}+2} \\
& \lambda_{3 \mathrm{p}+1} \frac{\prod_{\mathrm{s}=0}^{\mathrm{k}} \lambda_{3(\mathrm{p}+\mathrm{k})+1-3 \mathrm{~s}}}{\prod_{\mathrm{s}=0}^{\mathrm{k}} \lambda_{3(\mathrm{p}+\mathrm{k})+2-3 \mathrm{~s}}} \frac{\prod_{\mathrm{s}=0}^{2 \mathrm{k}+1} \lambda_{3(\mathrm{p}+2 \mathrm{k})+4-3 \mathrm{~s}}}{\prod_{\mathrm{s}=0}^{2 \mathrm{k}+1} \lambda_{3(\mathrm{p}+\mathrm{k})+5-3 \mathrm{~s}}}=\mathrm{b}_{3 \mathrm{p}+3}
\end{aligned}
$$

where the values $b_{3 p+2}$ and $b_{3 p+3}$ are just analogous to $b_{3 p+1}$.

By multiplying one obtains

$$
\mathrm{b}_{3 \mathrm{p}+1} \mathrm{~b}_{3 \mathrm{p}+2} \mathrm{~b}_{3 \mathrm{p}+3}=\lambda_{3 \mathrm{p}-1}^{3}
$$

and in this way we have obtained explicitly the unique completely mixed $E$-point in the game.

As a final remark we would like to say that in the case that the number of players is $3 \mathrm{k}$ one obtains difficult problems for solving it which we expect to consider in a further paper. 


\section{Bibliography}

(1945) Kaplansky, I.: A contribution of van Newmann's Theory of Games. Annals of Mathematics Vol. 46 pp. 474-479.

(1967) Marchi, E.: E-points for Games. Proceeding of National Academy of Sciences U.S.A. Vol. $57 \mathrm{~N}^{\circ} 4$ pp. 878-882.

(1990) -: On Equilibrium Points of Diagonal N-Person Games. JOTA Vol. 64, No 1.

(2004) -: E-Points of Diagonal Games I (submitted for publication).

(1989) Martínez, R.: Cálculo de E-points en juegos tripersonales. Doctoral Thesis Universidad Nacional de San Luis, San Luis, Argentina. 